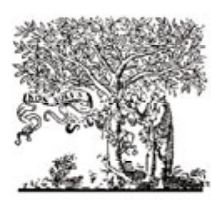

ELSEVIER
Available online at www.sciencedirect.com

ScienceDirect

Journal of Hydrodynamics

2017,29(5):906

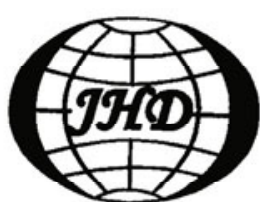

www.sciencedirect.com/ science/journal/10016058

\title{
32nd International Workshop on Water Waves and Floating Bodies (IWWWFB2017)
}

Dezhi Ning, Bin Teng

State Key Laboratory of Coastal and Offshore Engineering, Dalian University of Technology, Dalian 116023, China

The Thirty-Second Workshop on Water Waves and Floating Bodies was held in Dalian from April 23-26, 2017, and hosted by State Key Laboratory of Coastal and Offshore Engineering, Dalian University of Technology (DUT). IWWWFB2017 attracts over 90 participants from 17 countries, in which 65 participants from abroad. 57 papers were selected by the technical committee for presentation on the workshop and included in the proceeding from 80 submitted abstracts. The contributions cover a wide range of topics related to water waves and their effects on floating and submerged bodies. The presentations were divided into 15 sessions, and 25 minutes were arranged for each presentation to keep the workshop's regulation for creating the atmosphere of deeply discussion. The editorial board members of $<<$ Journal of Hydrodynamics $>>$, i.e., Profs. Dongqiang Lu, Decheng Wan, Changhong $\mathrm{Hu}$, Chiu-On $\mathrm{Ng}$, Yongwan Kim, Liang Cheng, Wenyang Duan and Dr. Xiaobo Chen, also attended the workshop and gave presentations. This workshop was financially supported by Bureau Veritas, K.C. WONG Education Foundation, State Key Laboratory of Coastal and Offshore Engineering and Department of Hydraulic Engineering of DUT, which made it possible to organize the workshop at a convenient place.

The International Workshop on Water Waves and Floating Bodies is an annual meeting of mathematicians and engineers with a particular interest in water waves and their effects on floating and submerged marine structures. The IWWWFB was initiated by Professor D. V. Evans (University of Bristol) and Professor J. N. Newman (MIT) following informal meetings between their research groups in 1984. First intended to promote communications between workers in UK and USA, the interest and participation quickly spread to include researchers from many other countries around the world. The workshop places particular emphasis on the participation of younger researchers, the stimulation of discussions between engineers and scientists, and the presentation of preliminary basic scientific work before its publication elsewhere.

The fundamental principle in the IWWWFB has been essentially the same since its inception. That is:

1. The focus of the workshop should be on water waves and their effects on floating and submerged bodies. Abstracts may be accepted in adjacent fields of specialty, provided they are sufficiently broad or profound to be of interest to this specific field.

2. Abstracts should be of high quality in that new ideas are included in the analytical and/or numerical work. Purely experimental work must emphasize underlying physics.

3. As far as these are satisfied, even incomplete and on-going work can be accepted.

4. Sufficient time for discussion will be provided not only just after each presentation but also after the session (during tea and coffee breaks, lunch, dinner etc.)

5. Attendance is restricted to the authors who submitted extended four-page abstracts. (Exceptions may exist for the past hosts, session chairpersons, and sponsors.)

The workshop is an important reference point for organizing and spreading knowledge in this area. In particular, the workshop proceedings are freely accessible through the dedicated internet address www.iwwwfb.org where all contributions from 1986 can be found. The 33rd IWWWFB will be held in L'Orient, France from April 4-7, 2018. 